Homology, Homotopy and Applications, vol. 9(2), 2007, pp.445-449

\title{
HOMOTOPY TYPES OF TRUNCATED PROJECTIVE RESOLUTIONS
}

\author{
W.H. MANNAN \\ (communicated by Graham Ellis)
}

\begin{abstract}
We work over an arbitrary ring $R$. Given two truncated projective resolutions of equal length for the same module, we consider their underlying chain complexes. We show they may be stabilized by projective modules to obtain a pair of complexes of the same homotopy type.
\end{abstract}

\section{Introduction}

Truncated projective resolutions are of interest in both algebraic geometry and algebraic topology. If the modules in a resolution of length $n$ are assumed to be free, then the $n^{\text {th }}$ homology group is the $n^{\text {th }}$ syzygy of the module being resolved. The minimal possible dimensions of the modules in such resolutions were of interest to mathematicians such as Hilbert and Milnor (see [2]).

In algebraic topology, truncated projective resolutions arise as the algebraic complexes associated to $(n-1)$-connected universal covers of CW-complexes of dimension $n$. Of particular interest is the case $n=2$, as classification of the homotopy types of these truncated resolutions is closely related to Wall's D2 problem (see the introduction to $[\mathbf{1}])$.

Given two truncated projective resolutions of the same module (of equal length), their final modules may be stabilized to produce homotopy equivalent algebraic complexes. This is a generalization of Schanuel's lemma which merely equates the final homology groups. The work of mathematicians such as Milnor, Whitehead and Wall suggests they were familiar with this basic homological result. Indeed, Wall's obstruction is suggestive of the modules required to stabilize the complexes (see $[\mathbf{3}], \S 3)$.

Given one truncated projective resolution of a module, this result provides a handle on all other possible truncated projective resolutions of the same length. Our purpose in this paper is to provide a simple proof of the result by explicitly constructing the desired homotopy equivalence between the two stabilized algebraic complexes.

Received June 20, 2007, revised October 19, 2007; published on November 12, 2007.

2000 Mathematics Subject Classification: 16E05.

Key words and phrases: projective resolution, homotopy type.

Copyright (c) 2007, International Press. Permission to copy for private use granted. 
Formally, let $R$ be a ring with identity and let $M$ be a module over $R$. We assume a right action on all modules. Suppose we have exact sequences:

$$
P_{n} \stackrel{\partial_{n}}{\longrightarrow} P_{n-1} \stackrel{\partial_{n-1}}{\longrightarrow} \cdots \stackrel{\partial_{2}}{\longrightarrow} P_{1} \stackrel{\partial_{1}}{\longrightarrow} P_{0} \stackrel{\epsilon}{\rightarrow} M-\rightarrow 0
$$

and

$$
Q_{n} \stackrel{\partial_{n}^{\prime}}{\longrightarrow} Q_{n-1} \stackrel{\partial_{n-1}^{\prime}}{\longrightarrow} \cdots \stackrel{\partial_{2}^{\prime}}{\longrightarrow} Q_{1} \stackrel{\partial_{1}^{\prime}}{\longrightarrow} Q_{0} \stackrel{\epsilon^{\prime}}{\rightarrow} M-\rightarrow 0
$$

with the $P_{i}$ and $Q_{i}$ all projective modules. Our main result is:

Theorem 1.1. The complexes

$$
P_{n} \oplus S_{n} \stackrel{\partial_{n} \oplus 0}{\longrightarrow} P_{n-1} \stackrel{\partial_{n-1}}{\longrightarrow} \cdots \stackrel{\partial_{2}}{\longrightarrow} P_{1} \stackrel{\partial_{1}}{\longrightarrow} P_{0}
$$

and

$$
Q_{n} \oplus T_{n} \stackrel{\partial_{n}^{\prime} \oplus 0}{\longrightarrow} Q_{n-1} \stackrel{\partial_{n-1}^{\prime}}{\longrightarrow} \cdots \stackrel{\partial_{2}^{\prime}}{\longrightarrow} Q_{1} \stackrel{\partial_{1}^{\prime}}{\longrightarrow} Q_{0}
$$

are chain homotopy equivalent, where the projective modules $T_{i}, S_{i}$ are defined inductively by $T_{0} \cong P_{0}, S_{0} \cong Q_{0}$ and for $i=1, \ldots, n, T_{i} \cong S_{i-1} \oplus P_{i}, S_{i} \cong T_{i-1} \oplus$ $Q_{i}$.

Given maps $f: A \rightarrow C, g: B \rightarrow C$, the notation $f \oplus g$ will always be used to denote the map $f \oplus g: A \oplus B \rightarrow C$ given by $f \oplus g:(a, b) \mapsto f(a)+g(b)$.

\section{Construction of chain homotopy equivalence}

For each $i \in 1, \ldots, n$ we have natural inclusions of summands:

$$
\iota_{i}: P_{i} \rightarrow T_{i}\left(\cong S_{i-1} \oplus P_{i}\right) \quad \iota_{i}^{\prime}: Q_{i} \rightarrow S_{i}\left(\cong T_{i-1} \oplus Q_{i}\right) .
$$

Let $\iota_{0}: P_{0} \rightarrow T_{0}$ and $\iota_{0}^{\prime}: Q_{0} \rightarrow S_{0}$ both be the identity map.

For $i=1, \ldots, n$, we define

$$
\begin{aligned}
\delta_{i}: T_{i}\left(\cong P_{i} \oplus S_{i-1}\right) & \rightarrow T_{i-1} \oplus S_{i-1} \\
\text { and } \delta_{i}^{\prime}: S_{i}\left(\cong Q_{i} \oplus T_{i-1}\right) & \rightarrow S_{i-1} \oplus T_{i-1}
\end{aligned}
$$

by

$$
\delta_{i}=\left(\begin{array}{cc}
\iota_{i-1} \partial_{i} & 0 \\
0 & 1
\end{array}\right) \quad \delta_{i}^{\prime}=\left(\begin{array}{cc}
\iota_{i-1}^{\prime} \partial_{i}^{\prime} & 0 \\
0 & 1
\end{array}\right)
$$

For $r=0, \ldots, n-1$, let $\mathcal{C}_{r}$ denote the chain complex

$$
P_{n} \oplus S_{n} \stackrel{\partial_{n} \oplus 0}{\longrightarrow} \cdots \stackrel{\partial_{r+2}}{\longrightarrow} P_{r+1} \stackrel{\iota_{r} \partial_{r+1}}{\longrightarrow} T_{r} \stackrel{\delta_{r}}{\longrightarrow} T_{r-1} \oplus S_{r-1} \stackrel{\delta_{r-1} \oplus 0}{\longrightarrow} \cdots \stackrel{\delta_{1} \oplus 0}{\longrightarrow} T_{0} \oplus S_{0} .
$$

Also let $\mathcal{C}_{n}$ denote the chain complex

$$
T_{n} \oplus S_{n} \stackrel{\delta_{n} \oplus 0}{\longrightarrow} T_{n-1} \oplus S_{n-1} \stackrel{\delta_{n-1} \oplus 0}{\longrightarrow} \ldots \stackrel{\delta_{2} \oplus 0}{\longrightarrow} T_{1} \oplus S_{1} \stackrel{\delta_{1} \oplus 0}{\longrightarrow} T_{0} \oplus S_{0} .
$$

Clearly $\mathcal{C}_{0}$ is the chain complex (1). For $r=0, \ldots n-1$, the chain complex $\mathcal{C}_{r+1}$ 
is obtained from $\mathcal{C}_{r}$ by replacing

$$
\stackrel{\partial_{r+2}}{\longrightarrow} P_{r+1} \stackrel{\iota_{r} \partial_{r+1}}{\longrightarrow} T_{r} \stackrel{\delta_{r}}{\longrightarrow}
$$

with

$$
\stackrel{\iota_{r+1} \partial_{r+2}}{\longrightarrow} P_{r+1} \oplus S_{r} \stackrel{\delta_{r+1}}{\longrightarrow} T_{r} \oplus S_{r} \stackrel{\delta_{r} \oplus 0}{\longrightarrow} .
$$

This is a simple homotopy equivalence, so $\mathcal{C}_{r+1}$ is chain homotopy equivalent to $\mathcal{C}_{r}$.

Similarly, for $r=0, \ldots, n-1$, let $\mathcal{D}_{r}$ denote the chain complex

$$
Q_{n} \oplus T_{n} \stackrel{\partial_{n}^{\prime} \oplus 0}{\longrightarrow} \cdots \stackrel{\partial_{r+2}^{\prime}}{\longrightarrow} Q_{r+1} \stackrel{\iota_{r}^{\prime} \partial_{r+1}^{\prime}}{\longrightarrow} S_{r} \stackrel{\delta_{r}^{\prime}}{\longrightarrow} S_{r-1} \oplus T_{r-1} \stackrel{\delta_{r-1}^{\prime} \oplus 0}{\longrightarrow} \cdots \stackrel{\delta_{1}^{\prime} \oplus 0}{\longrightarrow} S_{0} \oplus T_{0} .
$$

Again let $\mathcal{D}_{n}$ denote the chain complex

$$
S_{n} \oplus T_{n} \stackrel{\delta_{n}^{\prime} \oplus 0}{\longrightarrow} S_{n-1} \oplus T_{n-1} \stackrel{\delta_{n-1}^{\prime} \oplus 0}{\longrightarrow} \cdots \stackrel{\delta_{2}^{\prime} \oplus 0}{\longrightarrow} S_{1} \oplus T_{1} \stackrel{\delta_{1}^{\prime} \oplus 0}{\longrightarrow} S_{0} \oplus T_{0} .
$$

Clearly $\mathcal{D}_{0}$ is the chain complex (2). As before, for $r=0, \ldots n-1$, the chain complex $\mathcal{D}_{r+1}$ is chain homotopy equivalent to $\mathcal{D}_{r}$.

We have (1) chain homotopy equivalent to $\mathcal{C}_{n}$ and (2) chain homotopy equivalent to $\mathcal{D}_{n}$. We complete the proof of the theorem by showing that $\mathcal{C}_{n}$ is chain isomorphic to $\mathcal{D}_{n}$.

Lemma 2.1. There exist inverse pairs of maps $h_{i}, k_{i}$ making the following diagram commute:

$$
\begin{aligned}
& T_{n} \oplus S_{n} \stackrel{\delta_{n} \oplus 0}{\longrightarrow} T_{n-1} \oplus S_{n-1} \stackrel{\delta_{n-1} \oplus 0}{\longrightarrow} \ldots \stackrel{\delta_{2} \oplus 0}{\longrightarrow} T_{1} \oplus S_{1} \stackrel{\delta_{1} \oplus 0}{\longrightarrow} T_{0} \oplus S_{0} \stackrel{\epsilon \oplus 0}{\longrightarrow} M-\longrightarrow 0 \\
& \downarrow h_{n} \quad \downarrow h_{n-1} \quad \downarrow h_{1} \quad \downarrow h_{0} \quad \downarrow 1 \\
& S_{n} \oplus T_{n} \stackrel{\delta_{n}^{\prime} \oplus 0}{\longrightarrow} S_{n-1} \oplus T_{n-1} \stackrel{\delta_{n-1}^{\prime} \oplus 0}{\longrightarrow} \cdots \stackrel{\delta_{2}^{\prime} \oplus 0}{\longrightarrow} S_{1} \oplus T_{1} \stackrel{\delta^{\prime} \oplus 0}{\longrightarrow} S_{0} \oplus T_{0} \stackrel{\epsilon^{\prime} \oplus 0}{\longrightarrow} M-\longrightarrow 0 \\
& \downarrow k_{n} \quad \downarrow k_{n-1} \quad \downarrow k_{1} \quad \downarrow k_{0} \quad \downarrow 1 \\
& T_{n} \oplus S_{n} \stackrel{\delta_{n} \oplus 0}{\longrightarrow} T_{n-1} \oplus S_{n-1} \stackrel{\delta_{n-1} \oplus 0}{\longrightarrow} \ldots \stackrel{\delta_{2} \oplus 0}{\longrightarrow} T_{1} \oplus S_{1} \stackrel{\delta_{1} \oplus 0}{\longrightarrow} T_{0} \oplus S_{0} \stackrel{\epsilon \oplus 0}{\rightarrow} M-\rightarrow 0 .
\end{aligned}
$$

Proof. As $T_{0}, S_{0}$ are projective, we may pick $f_{0}, g_{0}$ so that the following diagrams commute:

$$
\begin{array}{ll}
T_{0} \stackrel{\epsilon}{\longrightarrow} M & T_{0} \stackrel{\epsilon}{\longrightarrow} M \\
\downarrow f_{0} \stackrel{\downarrow}{\longrightarrow} & \uparrow g_{0} \uparrow 1 \\
S_{0} \stackrel{\epsilon^{\prime}}{\longrightarrow} M & S_{0} \stackrel{\epsilon^{\prime}}{\longrightarrow} M .
\end{array}
$$

Define $h_{0}: T_{0} \oplus S_{0} \rightarrow S_{0} \oplus T_{0}$ and $k_{0}: S_{0} \oplus T_{0} \rightarrow T_{0} \oplus S_{0}$ by

$$
h_{0}=\left(\begin{array}{cc}
f_{0} & 1-f_{0} g_{0} \\
1 & -g_{0}
\end{array}\right) \quad k_{0}=\left(\begin{array}{cc}
g_{0} & 1-g_{0} f_{0} \\
1 & -f_{0}
\end{array}\right) .
$$

Direct calculation shows that $h_{0} k_{0}=1$ and $k_{0} h_{0}=1$. 
Homology, Homotopy and Applications, vol. 9(2), 2007

Also from commutativity of (3), we deduce:

$$
\left(\begin{array}{ll}
\epsilon^{\prime} & 0
\end{array}\right)\left(\begin{array}{cc}
f_{0} & 1-f_{0} g_{0} \\
1 & -g_{0}
\end{array}\right)=\left(\begin{array}{ll}
\epsilon^{\prime} f_{0} & \epsilon^{\prime}\left(1-f_{0} g_{0}\right)
\end{array}\right)=\left(\begin{array}{ll}
\epsilon & 0
\end{array}\right)
$$

and

$$
\left(\begin{array}{ll}
\epsilon & 0
\end{array}\right)\left(\begin{array}{cc}
g_{0} & 1-g_{0} f_{0} \\
1 & -f_{0}
\end{array}\right)=\left(\begin{array}{ll}
\epsilon g_{0} & \epsilon\left(1-g_{0} f_{0}\right)
\end{array}\right)=\left(\begin{array}{ll}
\epsilon^{\prime} & 0
\end{array}\right) .
$$

Hence the following diagrams commute:

$$
\begin{aligned}
& T_{0} \oplus S_{0} \stackrel{\epsilon \oplus 0}{\longrightarrow} M \quad T_{0} \oplus S_{0} \stackrel{\epsilon \oplus 0}{\longrightarrow} M \\
& \downarrow h_{0} \quad \downarrow 1 \quad \uparrow k_{0} \quad \uparrow 1 \\
& S_{0} \oplus T_{0} \stackrel{\epsilon^{\prime} \oplus 0}{\longrightarrow} M \quad S_{0} \oplus T_{0} \stackrel{\epsilon^{\prime} \oplus 0}{\longrightarrow} M .
\end{aligned}
$$

Now suppose that for some $0<i \leqslant n$, we have defined $h_{j}: T_{j} \oplus S_{j} \rightarrow S_{j} \oplus T_{j}$ and $k_{j}: S_{j} \oplus T_{j} \rightarrow T_{j} \oplus S_{j}$ for $j=0, \ldots, i-1$, so that for each $j$, we have $h_{j} k_{j}=1$ and $k_{j} h_{j}=1$. We proceed by induction.

As before, pick $f_{i}, g_{i}$ so that the following diagrams commute:

$$
\begin{array}{llrl}
T_{i} \stackrel{\delta_{i}}{\longrightarrow} T_{i-1} \oplus S_{i-1} & & T_{i} \stackrel{\delta_{i}}{\longrightarrow} T_{i-1} \oplus S_{i-1} \\
\downarrow f_{i} & \downarrow h_{i-1} & \uparrow g_{i} \quad \uparrow k_{i-1} \\
S_{i} \stackrel{\delta_{i}^{\prime}}{\longrightarrow} S_{i-1} \oplus T_{i-1} & S_{i} \stackrel{\delta_{i}^{\prime}}{\longrightarrow} S_{i-1} \oplus T_{i-1} .
\end{array}
$$

Define $h_{i}: T_{i} \oplus S_{i} \rightarrow S_{i} \oplus T_{i}$ and $k_{i}: S_{i} \oplus T_{i} \rightarrow T_{i} \oplus S_{i}$ by

$$
h_{i}=\left(\begin{array}{cc}
f_{i} & 1-f_{i} g_{i} \\
1 & -g_{i}
\end{array}\right) \quad k_{i}=\left(\begin{array}{cc}
g_{i} & 1-g_{i} f_{i} \\
1 & -f_{i}
\end{array}\right) .
$$

Direct calculation shows that $h_{i} k_{i}=1$ and $k_{i} h_{i}=1$.

Recall $h_{i-1} k_{i-1}=1$ and $k_{i-1} h_{i-1}=1$. From commutativity of (4) we deduce:

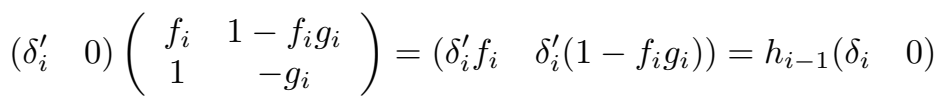

and

$$
\left(\begin{array}{ll}
\delta_{i} & 0
\end{array}\right)\left(\begin{array}{cc}
g_{i} & 1-g_{i} f_{i} \\
1 & -f_{i}
\end{array}\right)=\left(\begin{array}{ll}
\delta_{i} g_{i} & \delta_{i}\left(1-g_{i} f_{i}\right)
\end{array}\right)=k_{i-1}\left(\begin{array}{ll}
\delta_{i}^{\prime} & 0
\end{array}\right) .
$$

Hence the following diagrams commute:

$$
\begin{array}{rlrl}
T_{i} \oplus S_{i} \stackrel{\delta_{i} \oplus 0}{\longrightarrow} T_{i-1} \oplus S_{i-1} & T_{i} \oplus S_{i} \stackrel{\delta_{i} \oplus 0}{\longrightarrow} T_{i-1} \oplus S_{i-1} \\
\downarrow h_{i} & \downarrow h_{i-1} & \uparrow k_{i} & \uparrow k_{i-1} \\
S_{i} \oplus T_{i} \stackrel{\delta_{i}^{\prime} \oplus 0}{\longrightarrow} S_{i-1} \oplus T_{i-1} & S_{i} \oplus T_{i} \stackrel{\delta_{i}^{\prime} \oplus 0}{\longrightarrow} S_{i-1} \oplus T_{i-1} .
\end{array}
$$

So we may construct the $h_{i}, k_{i}$ as required.

We know the $h_{i}, i=0, \ldots, n$ constitute a chain map $h: \mathcal{C}_{n} \rightarrow \mathcal{D}_{n}$. Also the $k_{i}$ constitute a chain map $k: \mathcal{D}_{n} \rightarrow \mathcal{C}_{n}$. As $h$ and $k$ are mutually inverse we have that $\mathcal{C}_{n}$ and $\mathcal{D}_{n}$ are chain isomorphic. Hence (1) and (2) are chain homotopy equivalent as required. 
Homology, Homotopy and Applications, vol. 9(2), 2007

\section{Injective Resolutions}

Finally we note that dual arguments may be used in the same way to prove the dual result:

Theorem 3.1. Let $\left(I_{r}, \partial_{r}\right)$ and $\left(J_{r}, \partial_{r}^{\prime}\right)$ be injective resolutions for some module $M$, truncated after the $n^{\text {th }}$ terms $\left(\right.$ so $M \cong \operatorname{Ker}\left(\partial_{0}: I_{0} \rightarrow I_{1}\right) \cong \operatorname{Ker}\left(\partial_{0}^{\prime}: J_{0} \rightarrow J_{1}\right)$ ). Then stabilizing the final modules, $I_{n}$ and $J_{n}$, with the appropriate injective modules results in chain homotopy equivalent complexes.

\section{References}

[1] F.E.A. Johnson, Stable modules and the $D(2)$ problem, London Mathematical Society Lecture Note Series, Vol. 301, Cambridge University Press, Cambridge, 2003.

[2] J. Milnor, On the Betti numbers of real varieties, Proc. Amer. Math. Soc. 15 (1964), 275-280.

[3] C.T.C. Wall, Finiteness conditions for CW-complexes, Ann. of Math. (2), 81(1) (1965) 56-69.

W.H. Mannan wajid@mannan.info

Department of Pure Mathematics

University of Sheffield

Hounsfield Road

Sheffield S3 7RH

Great Britain

This article is available at http://intlpress.com/HHA/v9/n2/a16 